\title{
High Mobility Group Box 1 and Interleukin 6 at Intensive Care Unit Admission as Biomarkers in Critically III COVID-19 Patients
}

\author{
Chaisith Sivakorn, ${ }^{1 *}$ Jutamas Dechsanga, ${ }^{2}$ Lawan Jamjumrus, ${ }^{3}$ Kobporn Boonnak, ${ }^{4}$ Marcus J. Schultz, ${ }^{5,6,7}$ Arjen M. Dondorp, ${ }^{5,6,7}$ \\ Weerapong Phumratanaprapin, ${ }^{1}$ Ranistha Ratanarat, ${ }^{8}$ Thummaporn Naorungroj, ${ }^{8}$ Patchrapa Wattanawinitchai, ${ }^{3}$ \\ Tanaya Siripoon, ${ }^{1}$ Chatnapa Duangdee, ${ }^{9}$ and Tachpon Techarang ${ }^{10 \star}$ \\ ${ }^{1}$ Department of Clinical Tropical Medicine, Faculty of Tropical Medicine, Mahidol University, Bangkok, Thailand; ${ }^{2}$ Division of Pulmonary and Critical \\ Care, Department of Medicine, Chonburi Hospital, Chonburi, Thailand; ${ }^{3}$ Division of Pulmonary and Critical Care, Department of Medicine, \\ Buddhasothorn Hospital, Chachoengsao, Thailand; ${ }^{4}$ Department of Microbiology and Immunology, Faculty of Tropical Medicine, Mahidol \\ University, Bangkok, Thailand; ${ }^{5}$ Mahidol-Oxford Tropical Medicine Research Unit, Faculty of Tropical Medicine, Mahidol University, Bangkok, \\ Thailand; ${ }^{6}$ Department of Intensive Care \& Laboratory of Experimental Intensive Care and Anesthesiology (L.E.I.C.A), Academic Medical Center, \\ University of Amsterdam, Amsterdam, The Netherlands; ${ }^{7}$ Centre for Tropical Medicine and Global Health, Nuffield Department of Medicine, Oxford \\ University, Oxford, United Kingdom; ${ }^{8}$ Siriraj Hospital, Division of Critical Care, Department of Medicine, Faculty of Medicine, Mahidol University, \\ Bangkok, Thailand; ${ }^{9}$ Hospital for Tropical Disease, Faculty of Tropical Medicine, Mahidol University, Bangkok, Thailand; ${ }^{10}$ School of Medicine, \\ Walailak University, Nakhon Si Thammarat, Thailand
}

\begin{abstract}
Exuberant inflammation manifesting as a "cytokine storm" has been suggested as a central feature in the pathogenesis of severe coronavirus disease 2019 (COVID-19). This study investigated two prognostic biomarkers, the high mobility group box 1 (HMGB1) and interleukin-6 (IL-6), in patients with severe COVID-19 at the time of admission in the intensive care unit (ICU). Of 60 ICU patients with COVID-19 enrolled and analyzed in this prospective cohort study, 48 patients (80\%) were alive at ICU discharge. HMGB1 and IL-6 plasma levels at ICU admission were elevated compared with a healthy control, both in ICU nonsurvivors and ICU survivors. HMGB1 and IL-6 plasma levels were higher in patients with a higher Sequential Organ Failure Assessment (SOFA) score (>10), and the presence of septic shock or acute kidney injury. HMGB1 and IL-6 plasma levels were also higher in patients with a poor oxygenation status $\left(\mathrm{PaO}_{2} / \mathrm{FiO}_{2}<150 \mathrm{~mm}\right.$ $\mathrm{Hg}$ ) and a longer duration of ventilation (> 7 days). Plasma HMGB1 and IL-6 levels at ICU admission also correlated with other prognostic markers, including the maximum neutrophil/lymphocyte ratio, D-dimer levels, and C-reactive protein levels. Plasma HMGB1 and IL-6 levels at ICU admission predicted ICU mortality with comparable accuracy to the SOFA score and the COVID-GRAM risk score. Higher HMGB1 and IL-6 were not independently associated with ICU mortality after adjustment for age, gender, and comorbidities in multivariate analysis models. In conclusion, plasma HMGB1 and IL6 at ICU admission may serve as prognostic biomarkers in critically ill COVID-19 patients.
\end{abstract}

\section{INTRODUCTION}

Coronavirus disease 2019 (COVID-19) has emerged as a major threat worldwide, affecting more than 140 million people and resulting in more than 3 million deaths worldwide as of early April $2021 .^{1}$ The clinical course of COVID-19 varies substantially among patients. Most infected individuals remain asymptomatic or exhibit only mild to moderate symptoms; approximately $15 \%$ progress to severe pneumonia, and up to $5 \%$ may eventually need admission to the intensive care unit (ICU) due to acute respiratory distress syndrome (ARDS), shock, or multiple organ failure. ${ }^{2}$ A previous study suggested a scoring system (COVID-GRAM) predicting progress to critical illness, including admission to the ICU, requirement of invasive ventilation, and death in hospitalized COVID19 patients. ${ }^{3}$ Including inflammatory mediators might improve the prognostic models because of their central role in the pathogenesis of severe COVID-19. Exuberant inflammation manifesting as elevated levels of cytokines, commonly referred as a "cytokine storm" has been suggested to lead to critical conditions, such as ARDS, multiorgan failure, and eventually death.

The plasma level of high mobility group box 1 protein (HMGB1), one of the damage-associated molecular pattern molecules (DAMPs), has been found to correlate with excessive cytokine storm and severity of tissue damage in patients with severe

\footnotetext{
${ }^{*}$ Address correspondence to Chaisith Sivakorn, Department of Clinical Tropical Medicine, Faculty of Tropical Medicine, Mahidol University, Ratchathewi, Bangkok 10400, Thailand, E-mail: chaisith. siv@mahidol.edu or Tachpon Techarang, School of Medicine, Thasala, Nakhon Si Thammarat, 80161, Thailand, E-mail: g.tachpon@ gmail.com.
}

pneumonia or ARDS. ${ }^{4}$ HMGB1 initiates inflammation in COVID-19 patients via at least two separate pathways. The first pathway involves disulfide-HMGB1, triggering toll-like receptor-4, which causes the release of pro-inflammatory cytokines such as interleukin-6 (IL-6). The second pathway involves exogenous HMGB1, which induces the expression of the SARS-CoV-2 entry receptor (angiotensin-converting-enzyme-2 receptor) in alveolar epithelial cells in an advanced glycosylation end-product specific receptor dependent manner. ${ }^{5}$ In several murine models of pneumonia, targeting HMGB1 with monoclonal antibodies not only attenuates inflammatory lung injury but also decreases bacterial or viral burden in the lungs of mice. ${ }^{6,7} \mathrm{IL}-6$ is one of the main mediators of inflammatory and immune response initiated by infection or tissue injury, and increased plasma levels of IL- 6 have been reported in more than half of COVID-19 patients. ${ }^{8}$ One recent randomized clinical trial showed that an anti-IL-6 receptor antibody, tocilizumab, can prevent the need for invasive ventilation and death in COVID-19 patients. ${ }^{9}$

Early identification of inflammatory mediators predicting fatal or inferior outcomes may prompt early treatment interventions, potentially yielding better outcomes in patients with severe COVID-19. ${ }^{2}$ In this study, we aimed to define the prognostic roles of HMGB1 and IL-6 in critically ill patients with COVID-19.

\section{METHODS}

Design. The study protocol was reviewed and approved by the institutional review boards of the local ethics committees of Chonburi Hospital (129/63/S/h3) and Buddhasothorn Hospital (BSH-IRB 043/2563). Written informed consent was obtained from all patients or their first-line relative if the patient 
was too unwell to provide it under an emerging infectious disease provision.

Patients were treated following the Thai national COVID-19 treatment guideline from the Department of Disease Control. ${ }^{10}$ As a healthy control group, we analyzed stored healthy blood donors collected at pre-COVID-19 era with normal blood counts, normal values of liver enzymes, and a negative serology for viral hepatitis and HIV.

Patients. Sixty-seven critically ill COVID-19 patients were assessed for eligibility at admission to one of the participating ICUs, the Chonburi Hospital (Chonburi Province, Thailand) or the Buddhasothorn Hospital (Chachoengsao Province, Thailand), from January 1, 2020 through January 31, 2021. COVID-19 was confirmed by means of a positive SARS-CoV-2 real-time polymerase chain reaction assay of nasopharyngeal swabs, oropharyngeal swabs, or sputum samples. Patients were excluded if aged under 18 years or pregnant, or if admission was only for palliation. The remaining 60 COVID-19 patients were enrolled and included in the final analysis. Twelve patients (20\%) died in the ICU.

Blood sampling and measurements. Blood samples were prospectively collected at the time of ICU admission, before start of any antiviral treatment (including favipiravir, remdesivir, or lopinavir/ritonavir) or immunomodulators (which could be corticosteroids or IL-6 inhibitors). After centrifugation, plasma was stored at $-80^{\circ} \mathrm{C}$.

Plasma HMGB1 and IL-6 levels were determined using a commercial enzyme-linked immunosorbent assay (ELISA) kit according to the manufacturer's instructions (HMGB1 ELISA, cat. no. E-EL-H1554, Elabscience, Houston, TX; IL-6 ELISA, catalog number E-EL-H0102, Elabscience).

Laboratory data assessed daily in ICU included white blood cell count, hemoglobin, platelet count, absolute neutrophil and lymphocyte count, arterial blood gas analysis, total bilirubin levels, serum creatinine, D-dimer, C-reactive protein (CRP), lactate dehydrogenase (LDH), and lactate level .

Definitions. Sepsis was defined according to the Third International Consensus Definitions for Sepsis and Septic Shock (Sepsis-3) ${ }^{11}$ as an infection plus organ dysfunction identified by an increase in the Sequential Organ Failure Assessment (SOFA) score of 2 points or more. Septic shock was clinically identified by sepsis patients requiring vasopressor to maintain a mean arterial pressure of $65 \mathrm{~mm} \mathrm{Hg}$ or greater together with serum lactate level $\geq 2 \mathrm{mmol} / \mathrm{L}$ in the absence of hypovolemia.

ARDS was defined using the Berlin Definition ${ }^{12}$ in intubated COVID-19 patients or the Kigali Modification of the Berlin Definition $^{13}$ in nonintubated COVID-19 patients.

Metabolic acidosis was defined as a serum bicarbonate $<18$ $\mathrm{mmol} / \mathrm{L}$. Acute kidney injury (AKI) was diagnosed according to the Kidney Disease Improving Global Outcomes clinical practice guidelines. ${ }^{14}$ Hospital-acquired pneumonia (HAP) was defined as pneumonia that occurred 48 hours or more after admission and was not apparent at the time of admission. Ventilator-associated pneumonia was defined as pneumonia that presented more than 48 hours after endotracheal intubation.

Analysis plan. Values were expressed in numbers and proportions or means \pm SD where appropriate. Continuous variables were compared using Mann-Whitney $U$ test for nonnormal data distributions, and by independent $t$ test for normal data distributions. Dichotomous variables were compared using Pearson's $X^{2}$ test or Fisher's exact test.

Correlations between HMGB and IL-6 concentrations at ICU admission with peak laboratory data were estimated by Spearman's correlation. The ICU fatality prediction of HMGB levels, IL-6 levels, neutrophil/lymphocyte ratio, D-dimer level, SOFA scores, and COVID-GRAM risk scores

TABLE 1

Demographic and Clinical Characteristics of the ICU COVID-19 cohort

\begin{tabular}{|c|c|c|c|c|}
\hline Characteristics & Overall cohort $(N=60)$ & ICU nonsurvivors $(n=12)$ & ICU survivors $(n=48)$ & $P$ value \\
\hline \multicolumn{5}{|l|}{ Variables } \\
\hline Age, years & $46.3 \pm 14.1$ & $64.5 \pm 11.2$ & $41.7 \pm 10.7$ & $<0.001^{*}$ \\
\hline Sex, male/female & $31 / 29$ & $11 / 1$ & $20 / 28$ & $0.003^{*}$ \\
\hline BMI & $24.79 \pm 5.36$ & $25.94 \pm 6.71$ & $24.5 \pm 5$ & 0.407 \\
\hline \multicolumn{5}{|l|}{ Comorbidities, $n(\%)$} \\
\hline Chronic kidney disease & $15(25)$ & $8(66.7)$ & $7(14.6)$ & $0.001^{*}$ \\
\hline Hypertension & $15(25)$ & 7 (58.3) & $8(16.7)$ & $0.006^{*}$ \\
\hline Diabetes mellitus & $11(18.3)$ & $6(50)$ & $5(10.4)$ & $0.005^{\star}$ \\
\hline Stroke & $6(10)$ & $3(25)$ & $3(6.3)$ & 0.088 \\
\hline COPD/asthma & 2 (3.3) & $0(0)$ & $2(4.2)$ & NA \\
\hline \multicolumn{5}{|l|}{ COVID19 test reason, $n(\%)$} \\
\hline Contact with a confirmed COVID-19 case & $40(66.7 \%)$ & $12(100 \%)$ & $28(58.3 \%)$ & $0.005^{\star}$ \\
\hline Individual sought healthcare & $16(26.7 \%)$ & $0(0 \%)$ & $16(33.3 \%)$ & 0.025 \\
\hline Active surveillance & $3(5 \%)$ & $0(0 \%)$ & $3(6.3 \%)$ & NA \\
\hline Come back from other country & $1(1.7 \%)$ & $0(0 \%)$ & $1(2.1 \%)$ & NA \\
\hline \multicolumn{5}{|l|}{ COVID-19 treatment in ICU, $n(\%)$} \\
\hline Favipiravir & $55(91.7 \%)$ & $10(83.3 \%)$ & $45(93.8 \%)$ & 0.259 \\
\hline Remdesivir & $5(8.3 \%)$ & $3(25 \%)$ & $2(4.2 \%)$ & 0.050 \\
\hline Lopinavir/ritonavir & $49(81.7 \%)$ & $12(100 \%)$ & $37(77.1 \%)$ & 0.099 \\
\hline Hydroxychloroquine & $45(75 \%)$ & $10(83.3 \%)$ & $35(72.9 \%)$ & 0.712 \\
\hline Corticosteroid & $47(78.3 \%)$ & $8(66.7 \%)$ & $39(81.3 \%)$ & 0.271 \\
\hline IL-6 inhibitor (tocilizumab) & $9(15 \%)$ & $5(41.7 \%)$ & $4(8.3 \%)$ & 0.012 \\
\hline $\begin{array}{l}\text { Time from onset of illness to ICU } \\
\text { admission/laboratory measurements, } \mathrm{d}\end{array}$ & $9 \pm 2.86$ & $8+4.71$ & $9+2.7$ & 0.87 \\
\hline Length of ICU stay, d & $13.57 \pm 9.18$ & $20.25 \pm 13.37$ & $11.9 \pm 7.04$ & 0.057 \\
\hline
\end{tabular}


TABLE 2

Clinical parameters and laboratory findings of the COVID-19 cohort

\begin{tabular}{|c|c|c|c|c|}
\hline Characteristics & Overall cohort $(N=60)$ & ICU nonsurvivors $(n=12)$ & ICU survivors $(n=48)$ & $P$ value \\
\hline \multicolumn{5}{|l|}{ Clinical parameters } \\
\hline SOFA score at ICU admission & $7.6 \pm 5.24$ & $13.17 \pm 3.35$ & $6.21 \pm 4.69$ & $<0.001^{\star}$ \\
\hline Maximum SOFA score & $11.18 \pm 5.14$ & $17.25 \pm 2.22$ & $9.67 \pm 4.51$ & $<0.001^{\star}$ \\
\hline COVID-GRAM score at ICU admission & $129.21 \pm 35.73$ & $174.62 \pm 34.2$ & $117.85 \pm 25.9$ & $<0.001^{\star}$ \\
\hline \multicolumn{5}{|l|}{ ARDS severity at ICU admission } \\
\hline Mild $(200<\mathrm{PaO} 2 / \mathrm{FiO} 2 \leq 300 \mathrm{~mm} \mathrm{Hg})$ & $40(66.7 \%)$ & $0(0 \%)$ & 40 (83.3\%) & $<0.001^{\star}$ \\
\hline Moderate $(100<\mathrm{PaO} 2 / \mathrm{FiO} 2 \leq 200 \mathrm{~mm} \mathrm{Hg})$ & $3(5 \%)$ & $0(0 \%)$ & $3(6.3 \%)$ & NA \\
\hline Severe $(\mathrm{PaO} 2 / \mathrm{FiO} 2 \leq 100 \mathrm{~mm} \mathrm{Hg})$ & $17(28.3 \%)$ & $12(100 \%)$ & $5(10.4 \%)$ & $<0.001^{\star}$ \\
\hline \multicolumn{5}{|l|}{ Laboratory findings at ICU admission } \\
\hline Bilirubin (mg/dL) & $1.62 \pm 1.84$ & $4.14 \pm 2.82$ & $0.99 \pm 0.61$ & $0.003^{\star}$ \\
\hline $\mathrm{PaO} 2 / \mathrm{FiO} 2$ ratio $(\mathrm{mm} \mathrm{Hg})$ & $115.9 \pm 92.65$ & $92.67 \pm 9.07$ & $134.21 \pm 81.63$ & 0.025 \\
\hline Neutrophil/lymphocyte ratio & $12.62 \pm 4.87$ & $18.17 \pm 3.82$ & $11.24 \pm 4.07$ & $<0.001^{\star}$ \\
\hline Creatinine (mg/dL) & $2.26 \pm 1.22$ & $2.65 \pm 1.29$ & $2.04 \pm 1.17$ & 0.096 \\
\hline Hemoglobin (g/dL) & $10.78 \pm 2.3$ & $10.95 \pm 2.8$ & $10.35 \pm 2.2$ & 0.849 \\
\hline $\mathrm{LDH}(\mathrm{U} / \mathrm{L})$ & $385.83 \pm 140.2$ & $457.83 \pm 185.68$ & $367.83 \pm 122.27$ & 0.134 \\
\hline $\mathrm{CRP}(\mathrm{mg} / \mathrm{L})$ & $129.28 \pm 63.32$ & $154.25 \pm 40.42$ & $123.04 \pm 66.72$ & 0.128 \\
\hline D-dimer $(\mu \mathrm{g} / \mathrm{mL})$ & $2.47 \pm 1.19$ & $3.58 \pm 0.87$ & $2.19 \pm 1.1$ & $<0.001^{\star}$ \\
\hline Lactate $(\mathrm{mmol} / \mathrm{L})$ & $0.97 \pm 0.73$ & $1.31 \pm 0.54$ & $0.88 \pm 0.75$ & 0.068 \\
\hline \multicolumn{5}{|l|}{ Respiratory supports in ICU } \\
\hline HFNO & $37(61.7 \%)$ & $6(50 \%)$ & $31(64.6 \%)$ & 0.508 \\
\hline Noninvasive ventilation & $32(53.3 \%)$ & $2(16.7 \%)$ & $30(62.5 \%)$ & $0.008^{\star}$ \\
\hline Prone position & $28(46.7 \%)$ & $7(58.3 \%)$ & $21(43.8 \%)$ & 0.520 \\
\hline Invasive mechanical ventilation & $30(50 \%)$ & $11(91.7 \%)$ & $19(39.6 \%)$ & $0.003^{\star}$ \\
\hline \multicolumn{5}{|l|}{ Mechanical ventilation parameters in ICU } \\
\hline Plateau pressure $\left(\mathrm{cmH}_{2} \mathrm{O}\right)$ & $26 \pm 6$ & $30 \pm 4$ & $23 \pm 4$ & $<0.001^{\star}$ \\
\hline PEEP $\left(\mathrm{cmH}_{2} \mathrm{O}\right)$ & $11 \pm 2.96$ & $13.58 \pm 2.15$ & $9.37 \pm 2.11$ & $<0.001^{\star}$ \\
\hline Driving pressure $\left(\mathrm{cmH}_{2} \mathrm{O}\right)$ & $15.06 \pm 3.97$ & $16.83 \pm 3.76$ & $13.95 \pm 3.76$ & $0.046^{\star}$ \\
\hline Tidal volume (mL/PBW) & $8.08 \pm 0.89$ & $8.66 \pm 0.29$ & $7.71 \pm 0.94$ & $<0.001^{\star}$ \\
\hline Ventilator-free days & $7.52 \pm 4.2$ & $1.42 \pm 3.06$ & $9.04 \pm 2.84$ & $<0.001^{\star}$ \\
\hline \multicolumn{5}{|l|}{ Complications in ICU } \\
\hline Metabolic acidosis- $\left(\mathrm{HCO}_{3}^{-}<18 \mathrm{mmol} / \mathrm{L}\right)$ & $28(46.7 \%)$ & $12(100 \%)$ & $16(33.3 \%)$ & $<0.001^{\star}$ \\
\hline Pulmonary embolism & $5(8.3 \%)$ & $3(25 \%)$ & $2(4.2 \%)$ & 0.050 \\
\hline Septic shock & $21(35 \%)$ & $12(100 \%)$ & $9(18.8 \%)$ & $<0.001^{\star}$ \\
\hline HAP/NAP & $17(28.3 \%)$ & $8(66.7 \%)$ & $9(18.8 \%)$ & $0.002^{*}$ \\
\hline Renal replacement therapy & $6(10 \%)$ & $3(25 \%)$ & $3(6.3 \%)$ & 0.088 \\
\hline Pulmonary edema & $14(23.3 \%)$ & $6(50 \%)$ & $8(16.7 \%)$ & $0.024^{*}$ \\
\hline Acute kidney injury & $17(28.3 \%)$ & $12(100 \%)$ & $5(10.4 \%)$ & $<0.001^{\star}$ \\
\hline Maximum norepinephrine $(\mu \mathrm{g} / \mathrm{kg} / \mathrm{min})$ & $0.1 \pm 0.14$ & $0.27 \pm 0.2$ & $0.05 \pm 0.06$ & $<0.001^{\star}$ \\
\hline
\end{tabular}

at ICU admission were further evaluated by receiver operating characteristic curve (ROC) analysis, and areas under the curve (AUCs) were calculated. Youden Index was calculated on the basis of the ROC to help set the appropriate cutoff value as the best obtainable balance of sensitivity and specificity. Comparison of survival curves used Kaplan-Meier survival analysis, and differences were compared using the log-rank test. Multivariate analysis
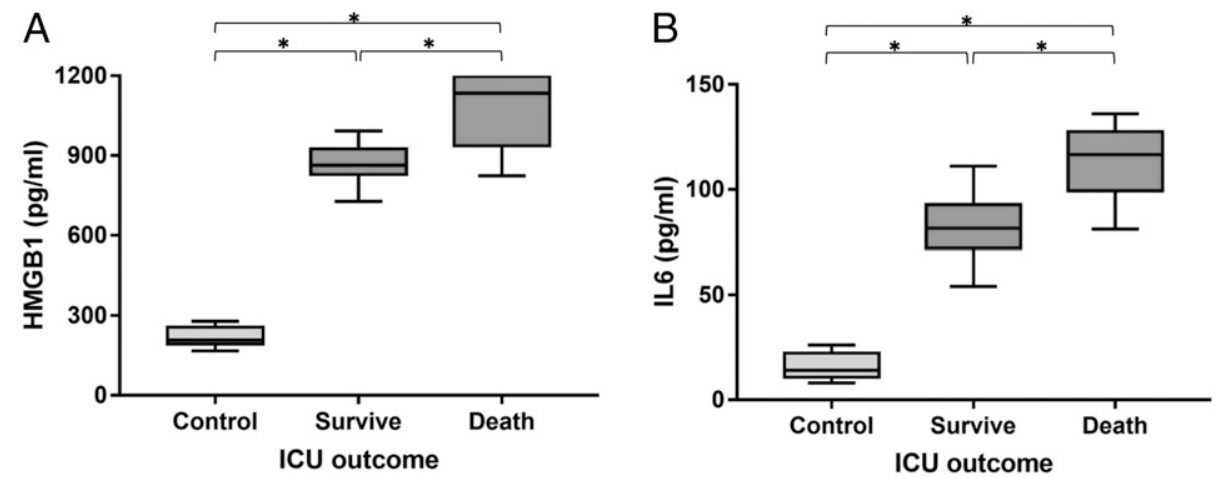

FigURE 1. Plasma was obtained from healthy control subjects (control; $N=20$ ), intensive care unit (ICU) nonsurvivors (death; $N=12$ ), ICU survivors (survive; $N=48$ ). (A, B) high mobility group box 1 (HMGB1) and interleukin-6 (IL-6) levels were elevated in death and survive group compared with control group, with an increase observed between death and survivor group. HMGB1 and IL-6 levels were highest in death COVID-19 patients. ${ }^{*} P<0.001$ ANOVA test with Bonferroni correction. 

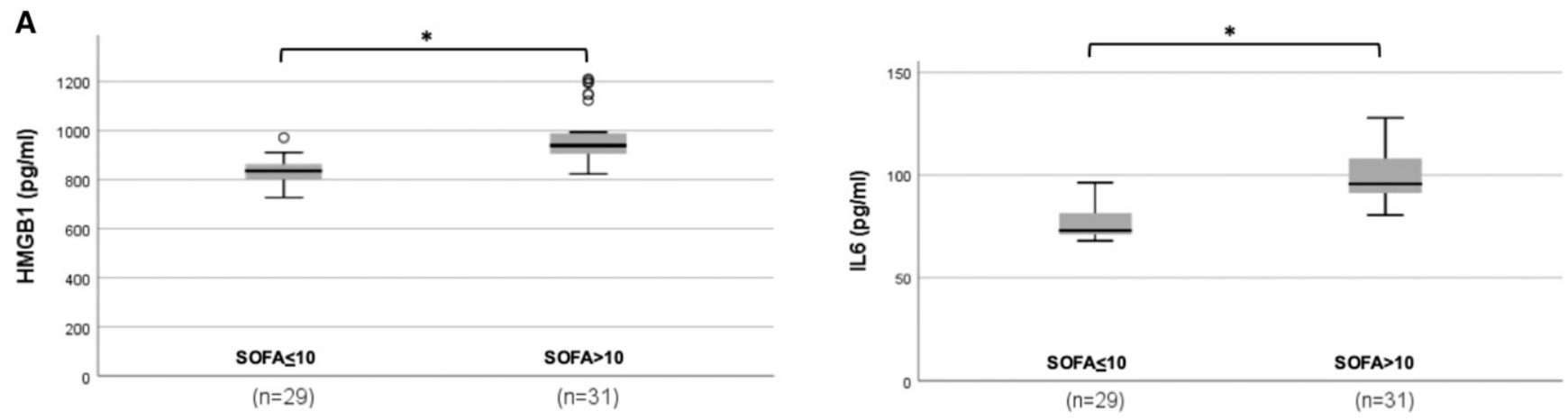

B

worst SOFA
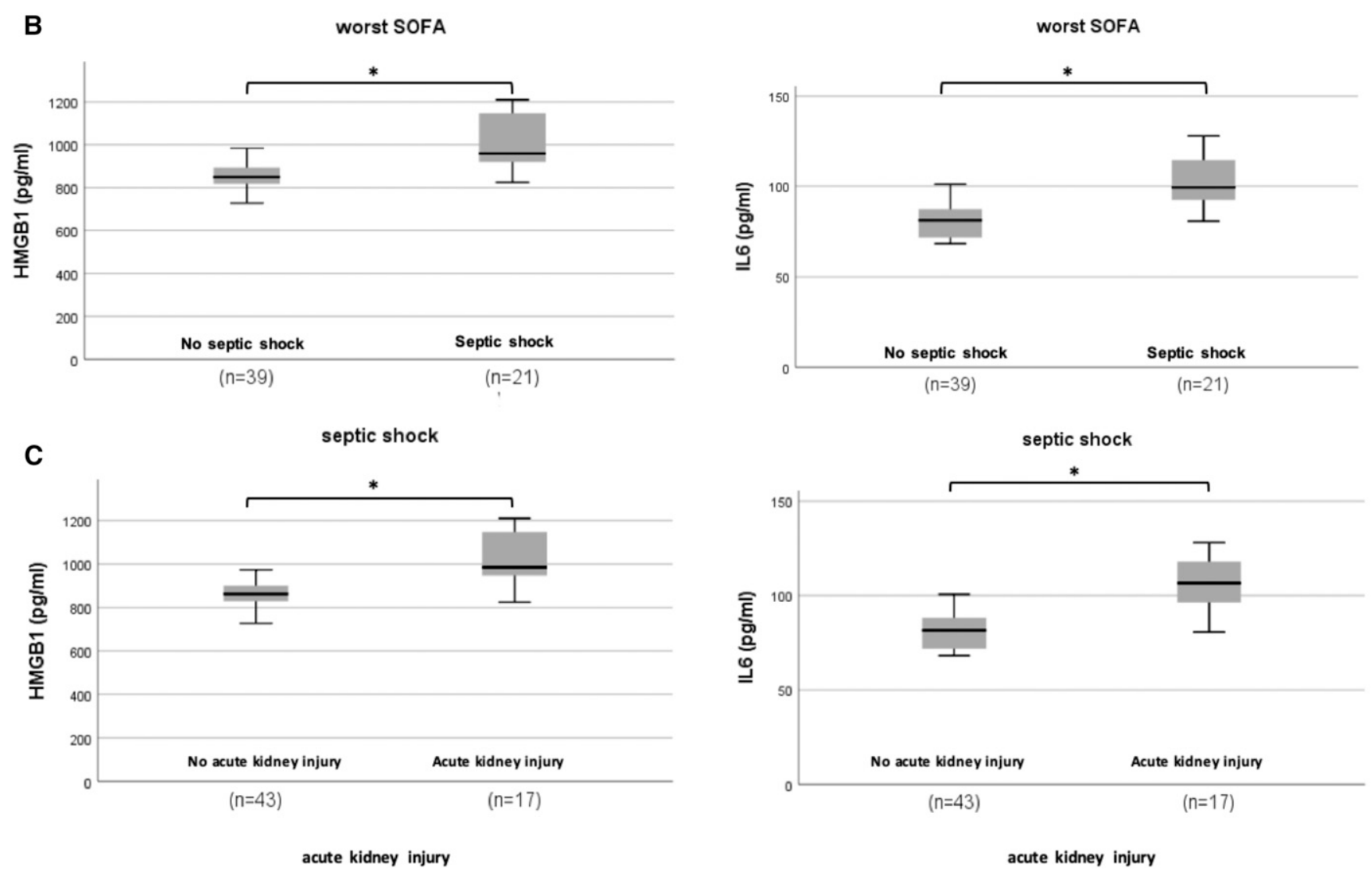

acute kidney injury

acute kidney injury

FIGURE 2. High mobility group box 1 (HMGB1) and interleukin-6 (IL-6) plasma levels in COVID-19 patients at intensive care unit (ICU) admission were significantly higher in ICU COVID-19 patients with (A) worse organ failure, as defined by a Sequential Organ Failure Assessment score > 10; (B), presence of septic shock; (C) presence of acute kidney injury; (D) a prolonged duration of mechanical ventilation, as defined by invasive mechanical ventilation use $>7$ days; $2 \mathrm{E}$, worse respiratory parameters, as defined by lower level of minimum PaO2/ FiO2 $(<150)$ in ICU. * $P<0.001$ Independent t-test.

was performed to adjust for disparate baseline characteristics contributing to mortality.

Data were analyzed using IBM SPSS Statistics for Windows version 18.0 (IBM Corp., Armonk, NY) and GraphPad Prism version 7.0 (GraphPad Software Inc., San Diego, CA).

\section{RESULTS}

Patients. Demographic and clinical characteristics are described in Table 1. ICU mortality was $20 \%$. Care for patients was not different for survivors versus nonsurvivors with regard to antiviral treatment; nonsurvivors received an IL-6 inhibitor more often.
Clinical parameters and laboratory results. ICU admission clinical parameters and laboratory findings are presented in Table 2. At ICU admission, nonsurvivors experienced worse clinical parameters including SOFA scores and COVID-GRAM scores. In addition, plasma bilirubin levels, the neutrophil/ lymphocyte ratio, and D-dimer levels were higher in nonsurvivors, whereas the $\mathrm{PaO}_{2} / \mathrm{FiO}_{2}$ ratio was higher in survivors.

During stay in ICU, nonsurvivors more often had severe ARDS and needed invasive ventilation more than survivors, with higher plateau pressure, positive end-expiratory pressure, driving pressure, and tidal volume. The number of ventilator-free days was lower in nonsurvivors, and metabolic 

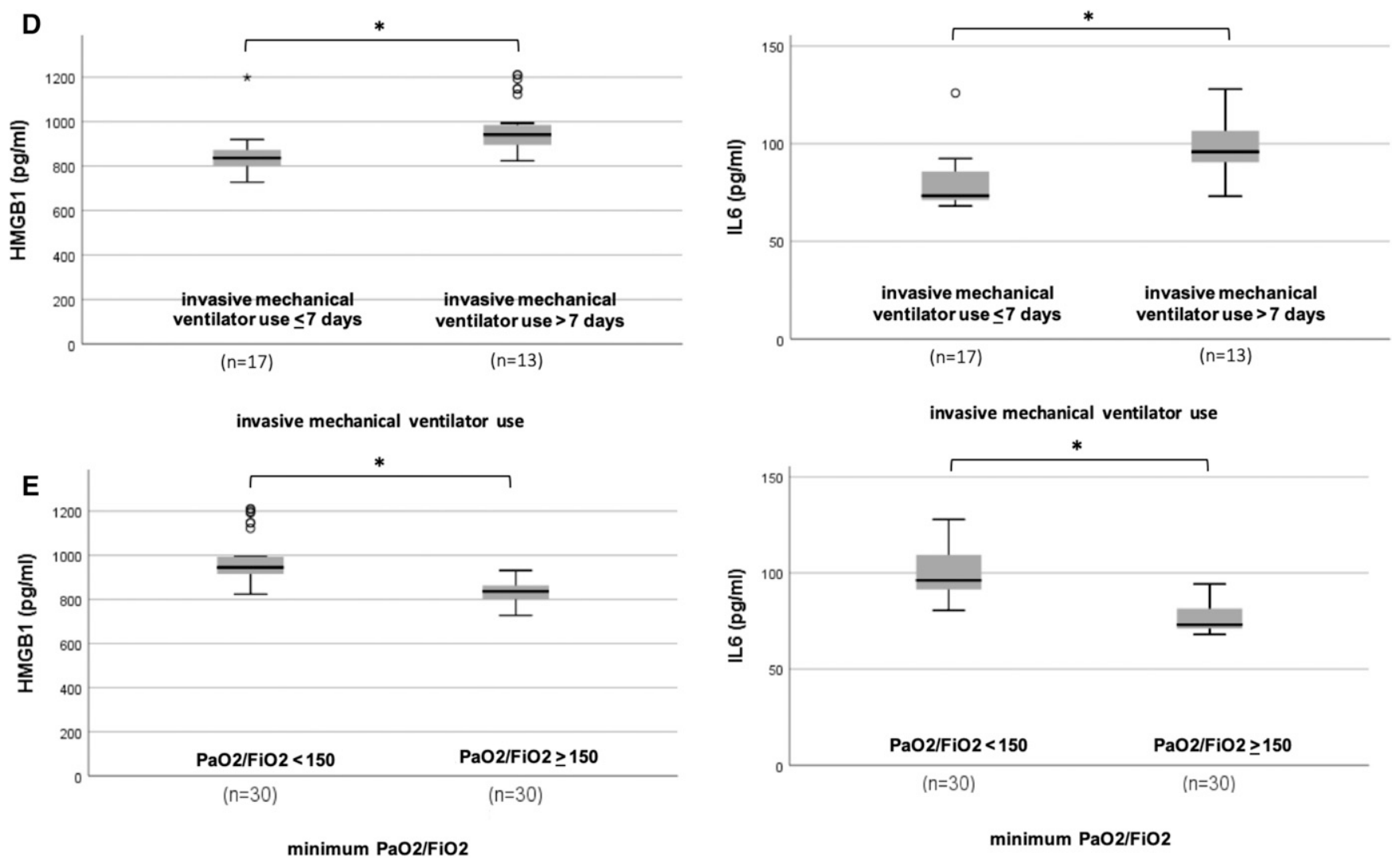

FIGURE 2. Continued.

acidosis, shock, pneumonia, pulmonary edema, and AKI was more often seen in nonsurvivors.

HMGB1 and IL-6 levels at ICU admission. HMGB1 and IL-6 levels were elevated in COVID-19 patients $(n=60)$ compared

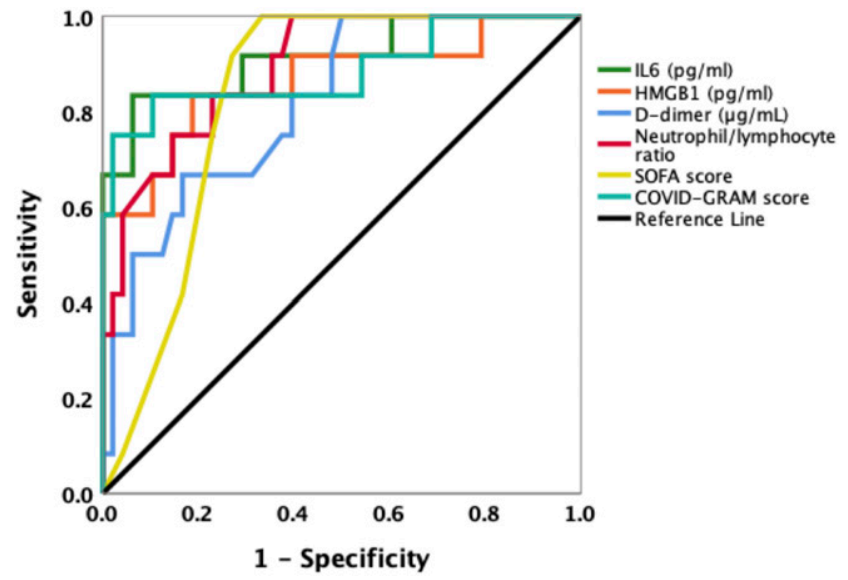

FIGURE 3. Receiver operating characteristic $(\mathrm{ROC})$ curves in predicting intensive care unit (ICU) survival based on interleukin-6 (IL-6) levels, high mobility group box 1 (HMGB1) levels, D-dimer levels, or neutrophil/lymphocyte ratio or Sequential Organ Failure Assessment (SOFA) score, or COVID-GRAM risk score at ICU admission in COVID19 patients. Areas under curves (AUCs) for IL-6 levels, HMGB1 levels, D-dimer levels, neutrophil/lymphocyte ratio, SOFA score, and COVIDGRAM risk score were $0.915,0.865,0.817,0.892,0.841$, and 0.885 , respectively. This figure appears in color at www.ajtmh.org. with healthy subjects (Figure 1 and Supplemental Table 1). HMGB1 levels (1065.2 \pm 142.79 versus $871.2 \pm 162.72 \mathrm{pg} / \mathrm{mL}$, $P<0.001)$ and IL-6 levels $(113.1 \pm 38.43$ versus $82.5 \pm 39.84$ $\mathrm{pg} / \mathrm{mL}, P<0.001$ ) were higher in nonsurvivors than in survivors.

HMGB1 and IL-6 levels correlated with all defined clinical outcomes during stay in ICU (Figure 2), and with peak neutrophil/lymphocyte ratios $\left(r_{\mathrm{s}}=0.375, P=0.038\right)$, peak CRP levels $\left(r_{s}=0.357, P=0.049\right)$ and peak $D$-dimer levels $\left(r_{s}=0.352\right.$, $P=0.006$ ) (Supplemental Table 2).

Prognostic value of HMGB1 and IL-6 plasma levels. The ROCs for HMGB1, IL-6, D-dimer, neutrophil/lymphocyte ratio, SOFA score, and COVID-GRAM risk score as a predictor for a fatal outcome in the ICU all showed high AUCs (Figure 3 and Supplemental Table 3 in Supplementary Information). The optimal cutoff for HMGB1 and IL-6 were $933.17 \mathrm{pg} / \mathrm{mL}$ and 97.83 $\mathrm{pg} / \mathrm{mL}$, respectively. Using these cutoffs, Kaplan-Meier analysis showed that patients with HMGB1 plasma levels $\geq 933.17$ $\mathrm{pg} / \mathrm{mL}$ or IL-6 plasma levels $\geq 97.83 \mathrm{pg} / \mathrm{mL}$ had higher ICU mortality (Figure 4). After adjustment for age, gender and comorbidities by multivariate analysis, higher levels of plasma HMGB1 and IL-6 at ICU admission were not independently associated with ICU mortality (Supplemental Table 4).

\section{DISCUSSION}

Within this cohort of severe COVID-19 patients admitted in ICU, HMGB1, and IL-6 plasma concentrations measured at ICU admission were highest in patients with a fatal course of the disease. Plasma concentrations of these biomarkers were 

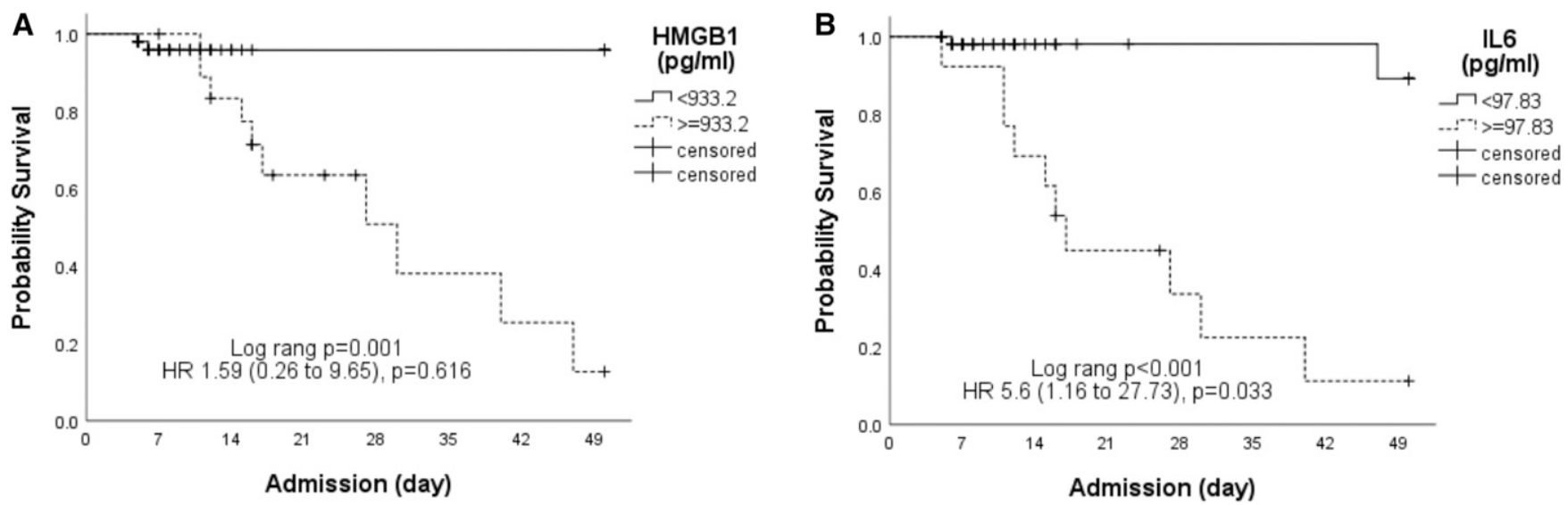

FIGURE 4. Kaplan-Meier survival curves of intensive care unit (ICU) COVID-19 patients are displayed, showing that patients with high mobility group box 1 (HMGB1) levels $\geq 933.2 \mathrm{pg} / \mathrm{mL}(P=0.001)$ (A) or interleukin-6 (IL-6) levels $\geq 97.83 \mathrm{pg} / \mathrm{mL}(P<0.001)(B)$ on ICU admission had a significantly higher ICU mortality compared with patients with HMGB1 levels $<933.2 \mathrm{pg} / \mathrm{mL}$ or IL-6 levels $<97.83 \mathrm{pg} / \mathrm{mL}$.

also higher in patients with a worse SOFA score (>10), septic shock, or AKI and were also associated with poor respiratory outcomes, including longer times on invasive mechanical ventilator ( $>7$ days) and a worse $\mathrm{PaO}_{2} / \mathrm{FiO}_{2}(<150)$. ROC curve analysis showed that HMGB1 and IL-6 plasma concentrations measured at ICU admission could predict subsequent death in ICU with high accuracy.

HMGB1, an important DAMP, can be actively released by innate immune cells in response to exogenous pathogens or endogenous inflammatory stimuli and can be passively released from damaged lung parenchymal cells. ${ }^{15-18}$ However, in contrast to classical inflammatory cytokines (e.g., tumor necrosis factor), in animal studies, HMGB1 is a late mediator of endotoxin lethality. ${ }^{19}$ Previous studies have shown increased HMGB1 plasma levels in critically ill patients ${ }^{20,21}$ but limited value as a prognosticator for death in the critically ill setting. ${ }^{20,22}$ This may have been caused by substantial heterogeneity in patient characteristics and inclusion of less severe patients compared with the present study. In COVID-19, it has been shown previously that HMGB1 plasma levels are elevated in severe disease, ${ }^{23,24}$ and are predictive of a fatal outcome, with an AUCs of $0.694 .{ }^{24}$ Our results are in accordance with these previous studies but show a substantial better accuracy for predicting a fatal outcome with an AUCs of 0.865 . This better discrimination could be explained by the later time point of HMGB1 assessment at the moment of ICU admission (median 9 days from disease onset), rather than the moment of hospital admission in the previous study. In line with the experimental sepsis studies, HMGB1 would thus be a late mediator of a potentially fatal dysregulated host response in CVID-19. HMGB1 triggers toll-like receptor-4, generating the release of pro-inflammatory cytokine including $\mathrm{LL}-6$, which is one of pathophysiological hallmarks of the cytokine storm in critically ill COVID-19 patients. ${ }^{25-27}$

IL-6 is well established as a biomarker for disease severity in COVID-1928-31 and as a predictor for adverse clinical outcomes including mortality, ${ }^{32-36}$ as well as for respiratory failure and the need for mechanical ventilation. ${ }^{37} \mathrm{IL}-6$ has been used as a prognostic biomarker in COVID-19-associated hyperinflammatory syndrome. ${ }^{38}$ In agreement with these studies, our results showed that IL-6 plasma levels at ICU admission were associated with disease severity in COVID-19 and were predictive of worse respiratory outcomes and mortality in ICU. The prognostic accuracy for a fatal outcome was higher compared with HMGB1 or D-dimer plasma concentrations, the neutrophil/lymphocyte ratio or SOFA or COVID-GRAM risk scores at admission to the ICU.

There was a significant correlation between both HMGB1 and IL-6 plasma levels at ICU admission and other prognostic laboratory biomarkers. Higher IL-6 plasma levels were associated with higher peak D-dimer and peak CRP in ICU, which can be explained by IL- 6 causing an increase acute phase reactants, such as C-reactive protein, fibrinogen, and hepcidin. ${ }^{39}$ In addition, the ratio of neutrophils to lymphocytes was positively correlated with IL-6 plasma levels but not with HMGB1, suggesting IL-6 plays a more important role in the substantial reduction of the peripheral lymphocytes associated with immunoparalysis in patients with COVID-19. ${ }^{36}$

Our results show that both HMGB1 and IL-6 are important biomarkers of disease severity in COVID-19 and could guide early recognition of the most severe patients at the moment of ICU admission. This could prompt increased monitoring and earlier treatment interventions, potentially yielding better disease outcomes.

The present study has limitations. First, HMGB1 and IL-6 are known to be increased in patients with acute ${ }^{40,41}$ or chronic kidney disease ${ }^{42}$ and diabetes, ${ }^{43,44}$ which are also conditions associated with mortality from COVID-19. This partly explains that plasma HMGB1 and IL-6 at ICU admission were not independently associated with ICU mortality after adjusting for baseline characteristics in the multivariate logistic regression model. Second, the HMGB1 and IL-6 plasma levels were prospectively measured at a single time point. A follow-up study with serial measurements and in a larger group of patients will be necessary to reevaluate the best prognostic cutoff values for HMGB1 and IL-6 and to confirm their prognostic value.

In summary the study showed that plasma concentrations of HMGB1 and IL-6 assessed at ICU admission could accurately identify COVID-19 patients with a fatal outcome of the disease, with a predictive precision similar to or better than the SOFA or the COVID-GRAM risk scores. Future studies should particularly focus on the practical clinical value of HMGB1 and IL-6, including developing a scoring system with plasma HMGB1 and IL-6 as biomarkers for early recognition of COVID-19 patients at risk for developing severe disease. In addition to IL-6, HMGB1 might also be a potential therapeutic target in severe COVID-19. 
Received February 10, 2021. Accepted for publication April 19, 2021.

Published online May 3, 2021.

Note: Supplemental appendices appear at www.ajtmh.org.

Acknowledgments: We thank all patients, staffs and medical technologists of Chonburi Hospital (Chonburi Province, Thailand) and Buddhasothorn Hospital (Chachoengsao Province, Thailand). The American Society of Tropical Medicine and Hygiene has waived the Open Access fee for this article due to the ongoing COVID-19 pandemic.

Financial support: The publication of this work was granted by Faculty of Tropical Medicine, Mahidol University, Bangkok, Thailand

Disclosure: The study protocol was reviewed and approved by the institutional review boards of the local ethics committees of Chonburi Hospital (129/63/S/h3) and Buddhasothorn Hospital (BSH-IRB 043/ 2563). The data that support the findings of this study are available from Chonburi and Buddhasothorn Hospital, but restrictions apply to their availability of these data and so are not publicly available. However, data are available from the authors upon reasonable request and with the permission of the institution.

Authors' addresses: Chaisith Sivakorn, Clinical Tropical Medicine, Mahidol University Faculty of Tropical Medicine, Bangkok, Thailand, E-mail: chaisith.siv@mahidol.edu. Jutamas Dechsanga, Division of Pulmonary and Critical Care, Department of Medicine, Chonburi Hospital, Chonburi, Chonburi, Thailand, E-mail: kratair_medicine@ hotmail.com. Lawan Jamjumrus and Patchrapa Wattanawinitchai, Division of Pulmonary and Critical Care, Department of Medicine, Buddhasothorn Hospital, Chachoengsao, Chachoengsao, Thailand, E-mails: lawanpang@gmail.com and ptchrpk@gmail.com. Kobporn Boonnak, Department of Microbiology and Immunology, Faculty of Tropical Medicine, Mahidol University, Salaya, Nakhon Pathom, Thailand, E-mail: kobporn.boo@mahidol.ac.th. Marcus J. Schultz, Intensive Care Unit, AMC, Amsterdam, Amsterdam, The Netherlands, E-mail: marcus.j.schultz@gmail.com. Arjen M. Dondorp, MahidolOxford Tropical Medicine Research Unit, Faculty of Tropical Medicine, Bangkok, Thailand, E-mail: arjen@tropmedres.ac. Weerapong Phumratanaprapin and Tanaya Siripoon, Department of Clinical Tropical Medicine, Faculty of Tropical Medicine, Mahidol University, Bangkok, Thailand, E-mails: weerapong.phu@mahidol.ac.th and tanaya.sir@mahidol.edu. Ranistha Ratanarat, Faculty of Medicine, Mahidol University, and Siriraj Hospital, Department of Medicine, Bangkok, Thailand, E-mail: ranittha@hotmail.com. Thummaporn Naorungroj, Mahidol University, Siriraj Hospital, Salaya, Nakhon Pathom, Thailand, E-mail: thummaporn.nao@mahidol.ac.th. Chatnapa Duangdee, Hospital for Tropical Disease, Mahidol University, Salaya, Nakhon Pathom, Thailand, E-mail: chatnapa.dua@mahidol.ac.th. Tachpon Techarang, School of Medicine, Walailak University, Thai Buri, Nakhon Si Thammarat, Thailand, E-mail: g.tachpon@gmail.com.

This is an open-access article distributed under the terms of the Creative Commons Attribution (CC-BY) License, which permits unrestricted use, distribution, and reproduction in any medium, provided the original author and source are credited.

\section{REFERENCES}

1. John Hopkins University, 2020. COVID-19 Dashboard by the Center for Systems Science and Engineering (CSSE). Available at: https://coronavirus.jhu.edu/map.html. Accessed May 1, 2021.

2. Cao X, 2020. COVID-19: immunopathology and its implications for therapy. Nat Rev Immunol 20: 269-270.

3. Liang $\mathrm{W}$ et al., 2020. Development and validation of a clinical risk score to predict the occurrence of critical illness in hospitalized patients with COVID-19. JAMA Int Med 180: 1081-1089.

4. Wang M, Gauthier A, Daley L, Dial K, Wu J, Woo J, Lin M, Ashby C, Mantell LL, 2019. The role of HMGB1, a nuclear damageassociated molecular pattern molecule, in the pathogenesis of lung diseases. Antioxid Redox Signal 31: 954-993.

5. Andersson U, Ottestad W, Tracey KJ, 2020. Extracellular HMGB1: a therapeutic target in severe pulmonary inflammation including COVID-19? Mol Med 26: 1-13.

6. Fink MP, 2014. HMGB1 as a drug target in staphylococcal pneumonia. Crit Care 18: 1-3.
7. Nosaka N, Yashiro M, Yamada M, Fujii Y, Tsukahara H, Liu K, Nishibori M, Matsukawa A, Morishima T, 2015. Anti-high mobility group box-1 monoclonal antibody treatment provides protection against influenza A virus (H1N1)-induced pneumonia in mice. Crit Care 19: 1-9.

8. Zhang Z-L, Hou Y-L, Li D-T, Li F-Z, 2020. Laboratory findings of COVID-19: a systematic review and meta-analysis. Scand $J$ Clin Lab Invest 80: 441-447.

9. Salama $C$ et al., 2020. Tocilizumab in patients hospitalized with COVID-19 pneumonia. N Engl J Med 384: 20-30.

10. Department of Disease Control, 2021. Guidelines for Health Care and Public Health Workers. Available at: https://ddc.moph.go.th/ viralpneumonia/eng/guideline_hcw.php. Accessed May 1, 2021.

11. Singer $M$ et al., 2016. The third international consensus definitions for sepsis and septic shock (Sepsis-3). JAMA 315: 801-810.

12. Force ADT, Ranieri V, Rubenfeld G, Thompson B, Ferguson N, Caldwell E, 2012. Acute respiratory distress syndrome. JAMA 307: 2526-2533.

13. Riviello ED et al., 2016. Hospital incidence and outcomes of the acute respiratory distress syndrome using the Kigali modification of the Berlin definition. Am J Respir Crit Care Med 193: 52-59.

14. Khwaja A, 2012. KDIGO clinical practice guidelines for acute kidney injury. Nephron Clin Pract 120: c179-c184.

15. Wang H, Zhu S, Zhou R, Li W, Sama AE, 2008. Therapeutic potential of HMGB1-targeting agents in sepsis. Expert Rev Mol Med 10: e32.

16. Chen G-Y, Tang J, Zheng P, Liu Y, 2009. CD24 and Siglec-10 selectively repress tissue damage-induced immune responses. Science 323: 1722-1725.

17. Andersson U, Tracey KJ, 2011. HMGB1 is a therapeutic target for sterile inflammation and infection. Annu Rev Immunol 29: 139-162.

18. Alpkvist H, Athlin S, Mölling P, Norrby-Teglund A, Strålin K, 2018. High HMGB1 levels in sputum are related to pneumococcal bacteraemia but not to disease severity in community-acquired pneumonia. Sci Rep 8: 1-9.

19. Wang H, Yang H, Czura CJ, Sama AE, Tracey KJ, 2001. HMGB1 as a late mediator of lethal systemic inflammation. Am J Respir Crit Care Med 164: 1768-1773.

20. Karlsson S, Pettilä V, Tenhunen J, Laru-Sompa R, Hynninen M, Ruokonen E, 2008. HMGB1 as a predictor of organ dysfunction and outcome in patients with severe sepsis. Intensive Care Med 34: 1046-1053.

21. Gaïni S, Koldkjær OG, Møller HJ, Pedersen SS, 2007. A comparison of high-mobility group-box 1 protein, lipopolysaccharidebinding protein and procalcitonin in severe community-acquired infections and bacteraemia: a prospective study. Crit Care 11: R76.

22. Yagmur E, Buendgens L, Herbers U, Beeretz A, Weiskirchen R, Koek GH, Trautwein C, Tacke F, Koch A, 2018. High mobility group box 1 as a biomarker in critically ill patients. J Clin Lab Anal 32: e22584.

23. Chen $\mathrm{R}$ et al., 2020. HMGB1 as a potential biomarker and therapeutic target for severe COVID-19. Heliyon 6: e05672.

24. Chen $L$ et al., 2020. Elevated serum levels of S100A8/A9 and HMGB1 at hospital admission are correlated with inferior clinical outcomes in COVID-19 patients. Cell Mol Immunol 17: 992-994.

25. Andersson $U$ et al., 2000. High mobility group 1 protein (HMG-1) stimulates proinflammatory cytokine synthesis in human monocytes. J Exp Med 192: 565-570.

26. Wang D et al., 2020. Clinical characteristics of 138 hospitalized patients with 2019 novel coronavirus-infected pneumonia in Wuhan, China. JAMA 323: 1061-1069.

27. Mo $P$ et al., 2020. Clinical characteristics of refractory COVID-19 pneumonia in Wuhan, China. Clin Infect Dis. Available at: https://doi.org/10.1093/cid/ciaa270.

28. Leisman DE et al., 2020. Cytokine elevation in severe and critical COVID-19: a rapid systematic review, meta-analysis, and comparison with other inflammatory syndromes. Lancet Respir Med 8: 1233-1244.

29. Cai $Q$ et al., 2020. COVID-19 in a designated infectious diseases hospital outside Hubei Province, China. Allergy 75: 1742-1752. 
30. Chen G et al., 2019. Clinical and immunologic features in severe and moderate forms of coronavirus disease. J Clin Invest 130: 2620-2629.

31. Sirivongrangson $P$ et al., 2020. Endotoxemia and circulating bacteriome in severe COVID-19 patients. Intensive Care Med Exp 8: 1-15.

32. Coomes EA, Haghbayan H, 2020. Interleukin-6 in COVID-19: a systematic review and meta-analysis. Rev Med Virol 30: 1-9.

33. Aziz M, Fatima R, Assaly R, 2020. Elevated interleukin- 6 and severe COVID-19: a meta-analysis. J Med Virol 92: 2283-2285.

34. Liu T, Zhang J, Yang Y, Ma H, Li Z, Zhang J, Cheng J, Zhang X, Zhao Y, Xia Z, 2020. The role of interleukin- 6 in monitoring severe case of coronavirus disease 2019. EMBO Mol Med 12: e12421.

35. Stukas S, Hoiland RL, Cooper J, Thiara S, Griesdale DE, Thomas $A D$, Orde MM, English JC, Chen LY, Foster D, 2020. The association of inflammatory cytokines in the pulmonary pathophysiology of respiratory failure in critically ill patients with coronavirus disease 2019. Crit Care Explor 2: e0302.

36. Chen LY, Hoiland RL, Stukas S, Wellington CL, Sekhon MS, 2020. Confronting the controversy: interleukin-6 and the COVID-19 cytokine storm syndrome. Eur Respiratory Soc 56: 2003006.

37. Herold T, Jurinovic V, Arnreich C, Lipworth B J, Hellmuth JC, von Bergwelt-Baildon M, Klein M, Weinberger T, 2020. Elevated levels of interleukin- 6 and CRP predict the need for mechanical ventilation in COVID-19. J Allergy Clin Immunol 146: 128-136.e4.
38. Webb BJ, Peltan ID, Jensen P, Hoda D, Hunter B, Silver A, Starr N, Buckel W, Grisel N, Hummel E, 2020. Clinical criteria for COVID19-associated hyperinflammatory syndrome: a cohort study. Lancet Rheumatol 2: e754-e763.

39. Tanaka T, Narazaki M, Kishimoto T, 2014. IL-6 in inflammation, immunity, and disease. Cold Spring Harb Perspect Biol 6: a016295.

40. Jones SA, Fraser DJ, Fielding CA, Jones GW, 2015. Interleukin-6 in renal disease and therapy. Nephrol Dial Transplant 30: 564-574.

41. Leelahavanichkul A, Huang $Y$, Hu X, Zhou H, Tsuji T, Chen R, Kopp JB, Schnermann J, Yuen PS, Star RA, 2011. Chronic kidney disease worsens sepsis and sepsis-induced acute kidney injury by releasing high mobility group box protein-1. Kidney Int 80: 1198-1211.

42. Bruchfeld A, Qureshi AR, Lindholm B, Barany P, Yang L, Stenvinkel P, Tracey KJ, 2008. High Mobility Group Box Protein-1 correlates with renal function in chronic kidney disease (CKD). Mol Med 14: 109-115.

43. Biscetti F, Rando MM, Nardella E, Cecchini AL, Pecorini G, Landolfi R, Flex A, 2019. High mobility group box-1 and diabetes mellitus complications: state of the art and future perspectives. Int J Mol Sci 20: 6258.

44. BowkerN, Shah RL, Sharp SJ, Stewart ID, Wheeler E, Ferreira MA, Baras A, Wareham NJ, Langenberg C, Lotta LA, 2020. Metaanalysis investigating the role of interleukin- 6 mediated inflammation in type 2 diabetes. EBioMedicine 61: 103062. 